\title{
MULTIFREQUENCY OBSERVATIONS OF THE JET OF 3C 273
}

\author{
H.-J. RÖSER, K. MEISENHEIMER and M. NEUMANN \\ Max-Planck-Institut für Astronomie, Königstuhl 17, D69117 Heidelberg, Germany \\ and \\ R.G. CONWAY \\ The Nuffield Astronomy Laboratories, Jodrell Bank, Macclesfield, Cheshire SK11 9DL, U.K.
}

Previously we have studied the jet of the quasar 3C 273 at optical and radio frequencies ([3], [1]). In our first set of X-ray data with $17.2 \mathrm{ksec}$ integration time obtained with the ROSAT HRI, the jet is easily visible extending out from the bright quasar core. The total number of counts in the jet lies in the range 200 to 300 , depending on the details of the background model . This corresponds to an X-ray flux $f_{\nu}\left(2.9 \times 10^{17} \mathrm{~Hz}\right)=65 \ldots 140 \mathrm{nJy}$ (lower limit, synchrotron radiation $\alpha=-0.8 \ldots$ upper limit, bremsstrahlung $\left.\alpha=0, \mathrm{NHI}=1.8 \times 10^{20} \mathrm{~cm}^{-2}\right)$, in good agreement with the value derived from the EINSTEIN observations [2].

Are the X-rays of synchrotron origin? From an extrapolation of the radio/optical spectra we expect synchrotron X-ray flux only from the inner region around knot A (13" from the core). All other parts show a synchrotron cutoff spectrum. The maximum level consistent with data from other wavebands would be $\approx 30 \mathrm{nJy}$ in the RosAT band, well below what is observed. Thus, to explain the observations as synchrotron emission would require a completely different component, with vanishing intensity at frequencies below the $\mathrm{X}$-ray regime.

The X-ray flux also exceeds the expected inverse Compton-scattered radiation both from the microwave background photons and from the quasar's own (isotropic) radiation field. Only a more contrived model (e.g. beaming) may be compatible with the observations. For a thermal bremsstrahlung scenario, densities of the order $1 \mathrm{~cm}^{-3}$ in a $5 \times 10^{7} \mathrm{~K}$ plasma are required, resulting in a mass of $\approx 10^{9} \mathrm{M}_{\odot}$ in a volume corresponding to a diameter of about $1^{\prime \prime}$. These values are difficult to reconcile with the very low rotation measure at radio wavelengths [1].

Thus, none of the straightforward emission models seems to reproduce the $\mathrm{X}$ ray properties and we have to think of a more refined picture to model this source.

\section{References}

1. R.G. Conway, S.T. Garrington, R.A. Perley, and J.A. Biretta. Synchrotron radiation from the jet of $3 \mathrm{C}$ 273. II. - The radio structure and polarization. Astron. Astrophys., 267:347-362, 1993.

2. D.E. Harris and C.P. Stern. X-ray emission associated with the jet in 3C 273. Astrophys. J., 313:136-140, 1987.

3. H.-J. Röser and K. Meisenheimer. The synchrotron light from the jet of 3C 273. Astron. Astrophys., 252:458-474, 1991.

T. J.-L. Courvoisier and A. Blecha: Multi-Wavelength Continuum Emission of AGN, 333-334.

(c) 1994 IAU. Printed in the Netherlands. 


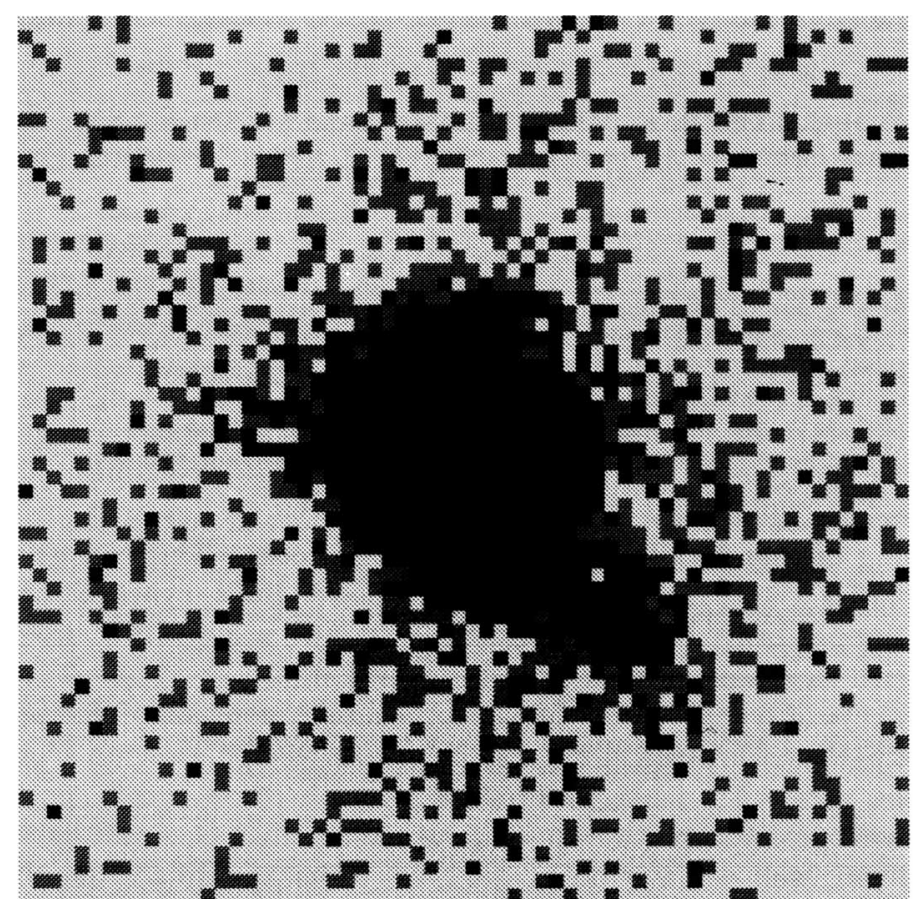

Fig. 1. Rosat HRI image of the quasar $3 \mathrm{C}$ 273. In comparison with the EINSTEIN image the jet is clearly visible at PA 222, extending out from the bright quasar core. The field shown here is $63^{\prime \prime} \times 63^{\prime \prime}$ in size and the HRI resolution is $2{ }^{\prime \prime} 5$. 